\title{
IMPLICACIÓN EMOCIONAL EN LA PRÁCTICA DE LA ENFERMERÍA
}

\author{
Sergio Romeo López Alonso \\ Diplomado Universitario en Enfermeria
}

\section{RESUMEN}

El presente trabajo discute la aptitud de las emociones en la práctica de la enfermería y expone diferentes argumentos desde los cuales el autor intenta extraer una conclusión, atendiendo a las relaciones interpersonales, a los resultados positivos de la implicación emocional en la práctica de la enfermería y a los riesgos potenciales que de esta implicación se podrían derivar, y por supuesto a la visión de los pacientes frente a esta.

Palabras clave: interrelación enfermero-paciente, antropologia clinica.

\section{SUMMARY}

The present work discusses about aptitude of the emotions in the nursing practice and exposes different arguments from which the author tries to draw a conclusion, considernig the interpersonal relations, the positive results of nursing's emotional implication and the potential risks that from this implication could be derived, and of course, the vision of the patients as opposed to this.

Keywords: nursing-patient interrelation, clinical anthropology

\section{I- INTRODUCCIÓN}

\section{I-1. REVISIÓN HISTÓRICA}

Desde su origen, las labores de enfermería en su periodo no profesional tuvieron que ser realizadas por la familia y los allegados del enfermo. En esta etapa de inexistencia de base científica, los cuidados intentaban cubrir todas las necesidades de los pacientes, aunque se centraban principalmente en los aspectos emocionales y espirituales, debido a los vínculos sentimentales que ligaban a estas personas con el paciente, a la escasez de conocimientos empíricos y a la atribución de las enfermedades a fuerzas sobrenaturales, tales como los dioses.

Posteriormente, y a pesar de la entrada de los conocimientos científicos en España, la evolución de los cuidados de enfermería siguió orientada a la satisfacción de las necesidades emocionales y espirituales, debido a la influencia del carácter vocacional y altruista impulsado por el cristianismo, que de algún modo demoraría la profesionalización de la enfermería.

Una vez conseguida esta profesionalización, la ciencia y la tecnología médica avanzaron muy deprisa, y la figura del prestador de cuidados se convirtió en asistente del médico, centrándose en los aspectos puramente físicos y técnicos de las enfermedades de los pacientes, dejando a un lado y en cierto sentido al conjunto de necesidades psicosociales y espirituales, que junto a las físicas se dan de manera integrada en el ser humano. Al mismo tiempo, la no implicación emocional estaba defendida por argumentos en los que se aseguraba la objetividad de la decisión médica, sin influencia de sentimientos que pudieran confundir la actuación de los profesionales de la salud. Además, con la entrada de Florence Nightingale en la enfermería, el concepto de buen enfermero implicaba tener la habilidad de ocultar las emociones y mantenerlas fuera del trabajo, no estando permitida la expresión de los sentimientos en estos profesionales. 
Hoy en día, la enfermería es una profesión con base científica, centrada en la prestación de cuidados para satisfacer todas y cada una de las necesidades básicas del ser humano, necesidades que van desde las biológicas hasta las espirituales, de forma que la salud posea un concepto holístico. Así, Maslow ${ }^{1}$ propuso las siguientes necesidades emotivas:

- Ser consciente de los propios sentimientos.

- Sentirse competente para expresar las propias emociones.

-Expresar libremente las propias emociones.

- Dar afecto.

- Recibir afecto.

- Ser sensible a las emociones de los otros. - Ser feliz.

\section{I-2. LA RELACIÓN INTERPERSONAL}

La relación interpersonal ${ }^{2}$ es la relación que cualquier sujeto desarrolla con otro. Es un proceso social que se caracteriza por su relativa estabilidad y viene determinado por la personalidad, la experiencia y las condiciones del medio.

No cabe duda de que la comunicación es soporte vital de esta relación y de que como fruto de ella nace la información. Ahora bien, la relación paciente-profesional de la salud y la información que de ella se deriva se hacen fundamentales en el proceso hacia la salud del paciente, proceso en el que el enfermero es el profesional que más oportunidades tiene de entablar esta relación. Por este motivo, el proceso hacia la salud va a depender en buen grado de esta relación.

Esta relación paciente-enfermero es la base del cuidado hacia otras personas, por lo que se convierte en uno de los requisitos indispensables para conseguir un cuidado humano, en otras palabras, un cuidado de enfermería profesional. Sobre el resultado de esta relación se basan una multitud de modelos de enfermería, como el de Hildgard Peplau, que definió la enfermería como un proceso interpersonal y terapéutico, en el que el enfermero trabaja cooperativamente con otro ser humano para posibilitar su salud. Por tanto, la enfermería tiene que cuidar sus estereotipos verbales y no verbales desde el primer contacto con el paciente; de hecho, la neutralidad afectiva ${ }^{3}$ es rara en la formación de una amistad o relación de ayuda, de esta manera se evitaría la confusión que podría llevar a este proceso fuera del camino hacia la salud.

Como consecuencia de estos encuentros, el enfermero va a obtener información, no sólo de la atención clínica, sino también de las actividades sociales diarias que forman parte del cuidado en el que ambos participan. Así, esta relación lleva al enfermero a una implicación emocional, que se constituye parte de su trabajo diario y que a la vez cumple unos objetivos clínicos y sociales, obtenidos a través de una serie de habilidades o técnicas de enfermería4.

\section{I-3. ¿CÓMO UNA RELACIÓN INTERPERSONAL SE CONVIERTE EN EMOCIONAL?}

La respuesta es fácil, como ya expusieron Walster Hatfield, Cacciopo \& Rapson, la razón radica en la existencia de un contagio emocional. Cuando una emoción es buena, por ejemplo, cuando un niño sonrie, no hay ningún problema, el enfermero también sonrie; pero las malas emociones pueden llevar al enfermero a una crisis, como

' Citado por Chalifur, J. 1994. La relación de ayuda en enfermeria, una perspectiva holistica. Barcelona: SG Editores.

${ }^{2}$ Novel Marti G., Lluch Canut, M.T. \& Miguel lopez de Vergara, M.D. 1995. Enfermeria psicosocial y salud menial. Barcelona: Masson S.A

${ }^{3}$ Novel Marti, Lluch Canut \& Miguel López de Vergara. Opus cit.

+ May, C. 1991 . Affective neutrality and involvement in nurse-patient relationships: perceptions of appropriate behaviour among nurses in acute medical and surgical ward. Journal of advanced nursing. 16(5): 552-8 
la muerte de un paciente. Es aquí donde se necesita un cierto control sobre las emociones. Por ello es útil saber que este contagio emocional no es fortuito o casual, sino que sigue un proceso de imitación y/o sincronía a través de una serie de mecanismos, entre los cuales se pueden resaltar:

-El proceso cognitivo consciente, donde se encuentran el análisis y la imaginación.

- La respuesta emocional condicionada e incondicionada, resultado de un proceso asociativo primitivo.

- La imitación y retroalimentación de la comunicación verbal y no verbal, y de los estados emocionales de otros.

Además, para que se dé un contagio emocional, la emoción contagiada debe reunir unas características que faciliten este evento, así pues:

- La emoción sentida debe ser o parecer muy fuerte. - La emoción debe ser expresada (con gestos faciales, verbal y/o posturalmente).

- Si existiera una emoción opuesta, la persona debe permanecer insensible o sin respuesta al sentimiento del otro.

Estas características son muy útiles para situar las emociones, tanto del paciente como del enfermero, dependiendo de la situación que se tenga que tratar.

Lo expuesto anteriormente podría servir también de base para una formación emocional con el fin de obtener ese grado de control que ayudara a cambiar las emociones negativas de los pacientes por una perspectiva más optimista y que al mismo tiempo evitara que los enfermeros llegaran a esos estados de crisis mental que pudiera interferir en su trabajo o afectar en detrimento de su situación emocional.

\section{I-4. LA IMPLICACIÓN EMOCIONAL COMO TRABAJO EMOCIONAL}

En el trabajo diario, la enfermería está expuesta a situaciones desagradables y molestas, muchas de las cuales son aún hoy en día temas tabú para la sociedad actual, como pueden ser el sufrimiento y la muerte. Sin embargo, conforme a Smith ${ }^{5}$, un enfermero tiene que modificar o suprimir sus propios sentimientos para conseguir que los pacientes se sientan cuidados y seguros, independientemente de lo que él sienta. Es entonces cuando se produce una diferencia entre las emociones sentidas y las expuestas al exterior, lo que implica un gasto extra de fuerza y energía, llamado trabajo emocional, de manera que mientras más intensa sea la emoción a la que se tienen que enfrentar, más duro será el trabajo emocional que se realice.

La dificultad para que este esfuerzo extra se considere trabajo radica en que el enfermero ofrece algo que normalmente no esta asociado con su propio trabajo. Cuántas veces se oye a un enfermero decir, mientras habla con un paciente, que deja la conversación porque tiene que hacer algo de trabajo (físico), para así justificar su empleo. Ambas labores requieren esfuerzo, y por tanto deben ser considerados como trabajo ${ }^{6}$.

\section{II- RAZÓN PARA LA IMPLICACIÓN EMOCIONAL}

\section{II.1- VALOR DEL TRABAJO EMOCIONAL}

Aunque desde hace años muchos autores han intentado situar y valorar la implicación emocional en el trabajo, fue James ${ }^{7}$ en 1989, y más tarde Staden en 1998, quienes afirmaron que aún se mantenía indefinida, inexplicada y, generalmen-

\footnotetext{
5 Smith, P. 1992. The emotional labour of nursing. London: Macmillan.

'Hochschild, 1983. Citado por Phillips, S. 1996. Labouring the emotions: expanding the remit of nursing work? Journal of advanced nursing. 24 (1): 139-43.

${ }^{7}$ Citado por Staden, H. 1998. Alertness to the needs of others: a study of the emotional labour of caring. Journal of advanced nursing. 27(1): 147-56.
} 
te, sin registrar; y que sólo era reconocida cuando iba mal o cuando el profesional ya no podía afrontarla durante más tiempo. Así, la dificultad para clarificar este término tiene la raíz en sí mismo.

No obstante, James vuelve a estudiar este término en 1992 y nos dice que, aunque encuentra difícil la definición de esta implicación emocional, la considera parte esencial del cuidado del paciente, que a su vez lo divide en: organización + trabajo físico + trabajo emocional, donde el trabajo físico es la estructura o soporte del emocional. Así pues, el trabajo de enfermería se encuentra dividido en tres partes tan importantes como necesarias, de manera que la prestación de un cuidado a un paciente estaría bien hecha cuando respondiera a todas estas partes, obteniendo así la satisfacción del paciente y del enfermero.

La satisfacción de necesidades y el sentimiento de ser cuidado es crucial. Los pacientes reconocen que los enfermeros trabajan emocionalmente, y eso es lo que esperan de ellos, que muestren alegría y felicidad, y que sepan enfrentarse a sentimientos extremos, independientemente de lo que como personas puedan sentir.

El trabajo emocional está constituido por la paciencia, el engatusamiento, la confrontación y otras muchas conductas empleadas por el enfermero para conseguir unos propósitos que forman parte del proceso de salud para los pacientes. Estas conductas son la realidad del continuo y arduo trabajo diario desempeñado por este profesional, conductas que en la mayoría de los casos son producto de la experiencia profesional y personal. De esta forma, el enfermero se convierte en una herramienta con dos funciones básicas para la salud de los pacientes, ya que además de saber cómo cuidar, también se implica y esfuerza para desarrollar este cuidado satisfactoriamente.

La importancia de definir la implicación emocional en el trabajo se basa en su reconocimiento y valoración, de manera que sea apoyado educativamente como parte integrante de los estudios de enfermería y de la posterior formación continuada, y organizativamente por parte de la gestión administrativa de los centros en los cuales dicho trabajo tiene lugar.

\section{II-2. EFECTOS BENEFICIOSOS DEL TRABAJO EMOCIONAL}

Bien es sabido que mientras más significativa sea una persona para otra, más posibilidades tendrá ésta de ganarse su confianza. En la vida cotidiana, la persona con la que tenemos más confianza coincide siempre con la que más contacto e implicación emocional tiene con nosotros. Si esto lo pasamos al campo asistencial para la salud, corresponde al enfermero ser la persona que más contacto tenga con el paciente, a lo que se añade también el conocimiento implícito del paciente sobre los estudios del enfermero, y si a esto se le suma una cierta implicación emocional, se logrará el nivel de confianza y entrega óptima para encauzar el proceso que llevará a la salud.

Esta confianza y entrega harán que el paciente se sienta relajado, seguro y lleno de fe respecto a los profesionales de la salud y al tratamiento que recibe de ellos, donde el enfermero tendrá un rol definido y satisfactorio, ejerciendo de pivote entre estos profesionales y los pacientes, obteniendo así una relación más eficiente y beneficiosa. De està manera se reduciría o eliminaría la tensión y el estrés, ayudando al paciente a comprender y actuar adecuadamente con su enfermedad, es decir, facilitando el autocuidado que se podría ver reflejado mayormente en la estricta adhesión al tratamiento y seguimiento de las consultas periódicas

Por otra parte, Phillips en 1996 apoyó lo que otros autores, como Strauss en 1982 y May en 1995, ya habían dicho al afirmar que el trabajo emocional ayuda a frenar las reacciones negativas de los pacientes, contrarrestándolas y dirigiéndolas, de forma que las provee de seguridad y de 
comprensión en torno al diagnóstico, pronóstico, examen y tratamiento. Asimismo genera un impacto en el bienestar psicológico y emocional del paciente, reduciendo sustancialmente el riesgo de síntomas y enfermedades psicosomáticas, como úlceras pépticas, arritmias, reducción del sistema inmunológico y otras; disminuyendo las complicaciones, y consecuentemente la estancia de hospitalización en su caso.

\section{II-3. ENFERMERO IDEAL PARA EL PACIENTE}

De acuerdo con la investigación llevada a cabo por $\mathrm{Smith}^{8}$, hay más de un $85 \%$ de pacientes que cuando intentan describir lo que ellos consideran como un buen enfermero, comienzan a hablar de actitudes y sentimientos, en vez de aptitudes técnicas. Los pacientes usan palabras como amabilidad, ayuda, paciencia, afectuosidad, alegría, cariño, consideración y animosidad, y también valoran las muestras de interés, escucha y charla por parte del enfermero, mientras que únicamente el 15\% restante hablaba sólo de eficiencia, observación, vigilancia y aptitud para realizar su trabajo. Una lectura de este resultado argumenta las expectativas fundamentales de los pacientes hacia el cuidado de enfermería, un cuidado distinto, aunque complementario al técnico, aportándole humanidad y haciendo más llevadero el camino, si es posible, hacia la salud, es decir, el alivio del malestar, dolor y sufrimiento mediante el soporte emocional.

Así Smith en 1992, al contemplar también el trabajo realizado por Hochschild en 1983 sobre la implicación emocional de las azafatas de vuelo, llegó a la conclusión de que los pacientes juzgan la calidad del servicio por el estilo emocional con que se $\mathrm{da}$, y su propia experiencia hace que valoren más el servicio sanitario por la calidad del cuidado emocional que por el técnico.
Este ideal del buen enfermero indica que de estos profesionales se espere mucho más de su entrega emocional que de su trabajo técnico. Irónicamente, los estudios de enfermería se centran mayormente en el campo físico-técnico. Al mencionar esto, y sin ánimo de subestimar la importancia de este último campo de estudio, al autor del artículo le gustaría enfatizar la importancia del terreno emocional, que complementa el campo físico-técnico, sujeto enteramente a la competencia de la enfermería, donde se le ofrece todo una línea de investigación y desarrollo.

\section{III- RIESGOS POTENCIALES DE LA IMPLICACIÓN EMOCIONAL}

La enfermería es una profesión donde la relación interpersonal paciente-enfermero es primordial y necesaria. Pero esta relación, aunque puede ser terapéutica si se emplean los mecanismos adecuados, no queda exenta de peligro. Por tanto, la calidad de esta relación será determinante para encontrar un equilibrio emocional, ya de por sí descompensado por la condición asimétrica de esta relación, que queda reflejada en sí misma, el que presta y el que recibe los cuidados.

\section{III-1. FUENTES DE DEMANDA EMOCIONAL}

Las fuentes que exigen una demanda emocional por parte de la enfermería son muchas y de muy diversa naturaleza, por estos motivos el autor de este artículo se va a centrar en las fuentes directas derivadas del contacto con el paciente y en algunas de las principales fuentes indirectas con clara influencia.

Así, según Rodríguez Marín, las fuentes directas más conocidas son:

- Sentimientos y comportamientos del paciente y familiares. La ansiedad, tristeza, frustración, ira, miedo... que son sólo algunos de los que la enfer- 
mería tiene que encarar y resolver satisfactoriamente.

-Naturaleza de la enfermedad. El pronóstico, deterioro biológico y psicológico, dependencia... que no son siempre prósperas y pueden determinar de alguna forma el estado mental de los enfermeros.

-Problema de comunicación. El paciente reservado, introvertido, tímido, el dar una mala noticia... que son caracteres y situaciones que también la enfermería tiene que confrontar y solventar de manera provechosa.

Aunque de forma menos frecuente, también se da la entrega excesiva de un enfermero a un paciente

De esta forma se suman una serie de actuaciones para las cuales la enfermería no ha sido preparada con una base científica para enfrentarse a ellas de forma eficaz.

En otra línea, aunque no con menos importancia, se sitúan las fuentes indirectas, entre las cuales se destacan:

-El cada vez más ajustado presupuesto.

- La tendencia a la reducción de plantilla.

- La gran cantidad de trabajo administrativo.

- La revolución tecnológica de los últimos años.

- La creciente demanda de conocimientos y de técnicas delegadas.

Todos estos factores provocan una división y organización desigual del trabajo de enfermería, así como del cuidado y la atención a los pacientes, que van a culminar no con una neutralidad emocional frente a él, sino con una implicación emocional inapropiada en perjuicio de la enfermería y de sus profesionales, y por consiguiente también del paciente.

\section{III-2. EFECTOS DE UNA IMPLICACIÓN EMO- CIONAL INADECUADA}

La enfermería, a la hora de tener una relación interpersonal con un paciente, debe establecer una implicación emocional acertada y unos mecanismos para mantenerla dentro de unos limites aceptables, ya que si dicha implicación se abre paso por caminos equivocados, va a desembocar en un deterioro de esta relación, y por tanto, el perjuicio será tanto para el enfermero como para el paciente.

El efecto más peligroso y conocido en este terreno es el Síndrome del quemado o Burnout syndrome. Este término, de acuerdo con muchos autores, fue acuñado por Freudemberg en 1974 , cuando lo definió como una de las consecuencias más preocupantes del estrés en el trabajo. Aunque serian Malash y Jackson en 1981 los que se aproximaron más al tema aquí tratado, cuando lo definieron como una respuesta inadecuada al estrés emocional crónico, que se caracteriza por un agotamiento físico y psicológico, una actitud distante y despersonalizada en la relación con otros, y un sentimiento de falta de adecuación al realizar sus tareas. Donde, según Schaufeli, Marek, \& Maslach, el agotamiento psicológico es una reducción de nuestros recursos emocionales y el sentimiento de no tener nada más que ofrecer psicológicamente a otros, la despersonalización es el desarrollo de actitudes negativas e insensibles y la falta de adecuación a las tareas puede estar enmascarada por actitudes de omnipotencia, surgidas de la amenaza de un sentimiento de incompetencia en el trabajo.

De los sintomas derivados del estrés que Álvarez Gallego y Fernández Ruiz encuentran más comunes, el autor de este artículo ha elegido:

-Psicosomáticos: Fatiga crónica, cefalea frecuente, insomnio, úlceras y otras alteraciones digesti-

' Citado por Ramos, J. Montalban, M. \& Bravo, M. 1997. Estrés y salud. Valencia: Promolibro.

"Citado por Llor, B., Abad, M.A., García, M. \& Nieto, J. 1996. Ciencias psicosociales aplicadas a la salud. Madrid: MacGraw-Hill Interamericana. 
vas, pérdida de peso, hipertensión, asma, dolor muscular (de espalda y cuello normalmente), reducción de la respuesta inmunológica, y periodo menstrual descontrolado en la mujer.

-Emocionales: Distancia afectiva enfermeropaciente, irritabilidad, rencor, incapacidad de concentración, baja autoestima, deseo de abandonar el trabajo -o la hospitalización en caso del paciente-, e incluso ideas suicidas.

$\mathrm{Y}$ ha desestimado los sintomas conductuales y defensivos, ya que los considera solamente como respuestas al estrés

En una visión general, según Alvarez Gallego y Femández Ruiz los enfermeros que sufren el síndrome del quemado presentan:

-Falta de energía y entusiasmo, no encuentran ninguna recompensa en la enfermería, y muestran un bajo interés en sus pacientes a los que percibe como fuente de frustración

-Falta de motivación en lo que hacen. Los enfermeros paran de mirar al futuro dentro de la profesión y comienzan a buscar nuevos intereses diferentes a la enfermería.

Por lo que la prestación de cuidados se vuelve mecánica y rutinaria, no hay individualización del cuidado, se comienza a etiquetar y estereotipar pacientes, y a centrarse exclusivamente en el trabajo físico dejando a un lado la visión holística del cuidado.

Todo sufrimiento emocional causado mientras los enfermeros sufren este sindrome acaba en un detrimento en la recuperación del paciente, prolongando su estancia en el hospital debido a complicaciones y/o a un proceso más lento de convalecencia. De manera que los pacientes empiezan a adoptar un sentimiento reacio hacia la hospitalización y a los profesionales de la salud.

Aparte de los problemas desatados por el estrës, también existen otros problemas derivados de una implicación inadecuada que afectan al cuidado del paciente, de los cuales cabe resaltar:
-Paciente demasiado dependiente de un enfermero, hasta producir un sentimiento de soledad en el paciente si el enfermero no está presente, por lo que puede incluso competir con otros pacientes para obtener la atención del enfermero. Según May, el paciente también se puede sentir muy ligado a un enfermero y no expresar lo que siente al resto de los enfermeros.

-Pérdida de objetividad de la condición de un paciente por parte del enfermero debido a la imposición de las emociones sobre la razón.

\section{III-3. RESPUESTA AL ESTRÉS}

De acuerdo con Chalifur, los enfermeros, al igual que los pacientes o cualquier otra persona, cuando se sienten bajo los efectos del estrés, implementan una serie de diferentes mecanismos para reducir o vencer este sentimiento de malestar.

Estos mecanismos o reacciones pueden ser conscientes o inconscientes, atendiendo a la voluntad de la persona, que para combatir al estrés recurre a ellos. No obstante, por cuestión de transcendencia y proximidad a la realidad cotidiana sólo se van a mencionar los mecanismos conscientes.

Estas respuestas șon fáciles de identificar, pretenden proteger el nivel de perturbación causado por el estrés y la ansiedad. Por este motivo, los enfermeros podrán verse competentes mientras afrontan la fuente de estrés, utilizando una conducta que podrá ser destructiva, como la hostilidad, la agresividad y la culpa, o constructiva a través de un proceso que lleva a la solución del problema; esta última va generalmente acompañada por una actitud de autoaceptación y respeto hacia los otros.

En el extremo opuesto, podrán situarse los enfermeros que decidan eludir la situación estresante simplemente con la evitación, aquí el absentismo será el gran protagonista, sin embargo estos comportamientos irán acompañados de sentimientos de frustración, miedo y vergüenza, que 
serán en último caso la aceptación del error; al mismo tiempo que el enfermero podrá sentir la hostilidad de sus compañeros si estos descubrieran su situación, desencadenando así un peor estado emocional.

En un punto intermedio se encontraría el compromiso, el retraso en la satisfacción de una necesidad, la aceptación de una satisfacción de nivel menor, o la modificación de los objetivos; de manera que el enfermero destacaría los objetivos obtenidos para hacer balance con la deficiencia de los que no ha conseguido; y así sobrellevar más fácilmente la situación de angustia y/o estrés que siente.

\section{IV- CONCLUSIÓN Y DISCUSIÓN}

Este artículo pone de relieve el importante papel que la implicación emocional juega en el trabajo diario de la enfermería, en el que se observa la imposibilidad de elección cuando el cuidado del paciente se hace de forma holística, es decir, una enfermería profesional y por tanto con base científica orientada hacia el ser humano.

Por otra parte, a pesar del riesgo que conlleva dicha implicación, al tener conciencia de los múltiples efectos beneficiosos que de este trabajo imocional se derivan, el autor de este artículo, al igual que el resto de autores que proponen la curazión favorecida por este medio, no duda en afirmar que merecerá la pena, siempre que de ella resulte una mayor satisfacción del paciente.

Por este motivo, carecería de sentido cualquier objeción ante el establecimiento de una aprojiada asignatura en los estudios de enfermería y somo parte de la formación continuada, la cual somprenda todo lo necesario que un enfermero leba saber sobre el trabajo emocional en su prácti:a profesional, para obtener ese grado de control iobre las situaciones emocionales oportunas. jiguiendo el trabajo de Smith en 1992, se podría concluir que el tema principal de esta asignatura estaría basado en el desarrollo de técnicas de comunicación y de comprensión psicológica, que permitieran la priorización y reflexión sobre las necesidades psicosociales en pacientes, y sobre el apoyo a los estudiantes y/o profesionales de la salud.

El reto que se expone sería una flexibilidad emocional con la que el enfermero combine una adecuada implicación emocional y un control profesional de estas emociones, que hiciera posible la carga y descarga de emociones tanto del paciente como del enfermero; y que dicha implicación se comporte como un epifenómeno, es decir, que los problemas o efectos secundarios surgidos del trabajo emocional no causen ninguna influencia sobre el proceso de cuidados de enfermería.

Parece digna de mención, la investigación llevada a cabo por Hochschild ${ }^{11}$ en 1983 sobre la implicación emocional que las azafatas de vuelo realizan en su trabajo, y también sobre la formación emocional que reciben. Esto no hace sino resaltar aún más la lamentable situación de los estudios de enfermería desprovistos de preparación emocional, si se considera que las azafatas tratan mayormente con gente sana y feliz, y los enfermeros tratan con gente psicológicamente angustiada.

Así pues, para que este aprendizaje emocional no caiga en la utopía, Daniel Goleman ofrece una serie de habilidades a desarrollar para obtener el control emocional preciso, y como muestra de su eficacia expone varios proyectos realizados con éxito en América, como son: Child Development Project en California y Seattle Social Development Project en Washington, entre otros.

Por otra parte, como resistencia principal a este trabajo emocional y como factor determinante de los cuidados se encuentra la cultura de una economía centrada en el ahorro de dinero, con la minimización de plantilla, con los enfermeros 
haciendo trabajos administrativos, con la falta de recursos materiales... pero esta actuación resulta contraria a su ideal. Respecto a esto, Goleman afirma que existe evidencia de una mejor y más rápida convalecencia en pacientes que han sido cuidados holísticamente; en otras palabras, emocional y físicamente en conjunto, pero esto es un resultado a medio y largo plazo, y los sistemas de gestión vigentes no poseen la paciencia necesaria para permitir esta realidad.

\section{V- BIBLIOGRAFÍA}

ALDRIDGE, M. 1994. Unlimited liability? Emotional labour in nursing and social work. Journal of advanced nursing. 20(4): $772-8$.

Áivarez Gallego, E. Ex Fernández Ríos, L. 1991. El síndrome de burn-out o el desgaste profesional. Revisión de estudios. Revista de la Asociación Española de Neuropsiquiatria. 39, 257-265.

Chalifur, J. 1994. La relación de ayuda en enfermería, una perspectiva holistica. Barcelona: Edit. SG Editores.

COULEHAN, J.L. 1995. Tenderness and steadiness. Literature and medicine. 14(2):222-36.

JAMES, N. 1992. Care $=$ organisation + physical work + emotional labour. Sociology of health \& illness. 14(4): 488-509.

Llor, B., Abad, M.A., Garcia, M. \& Nieto, J. 1996. Ciencias psicosociales aplicadas a la salud. Madrid: MacGraw-Hill Interamericana.

GILLAN, J. 1994. The emotional void. Nursing times. 90(10): 54 . GOLEMAN, D. 1997. 15. a edition. Inteligencia Emocional. Barcelona: Kairos, Colección ensayo.

MAY, C. 1991. Affective neutrality and involvement in nursepatient relationships: perceptions of appropriate behaviour among nurses in acute medical and surgical ward. Journal of advanced nursing. 16(5): 552-8.
NiTSCHKE, S.D. 1990. Is "love" a four letter word in additions nursing... What's wrong with "loving" our addicted clients?. Additions Nursing Network. 2(2):11-2,14.

Novel Marti G., Lluch Canut, M.T. \& Miguel López de VERGARA, M.D. 1995. Enfermeria psicosocial y salud mental. Barcelona: Editorial Masson S.A.

PAECH, M. 1996. Emotional labour of nurses. Contemporary nurse. 5(1):22-25

Peplau, H. 1990. Relaciones interpersonales en enfermería. Barcelona. Edit. Masson-Salvat.

PHILLIPS, S. 1996. Labouring the emotions: expanding the remit of nursing work? Journal of advanced nursing. 24(1): 13943.

Ramos, J. Montalbán, M. \& Bravo, M. 1997. Estrés y salud. Valencia: Promolibro

Rodríguez-Marin, J. 1995. Psicologia social de la salud. Madrid: Sintesis.

Schaufeli, W., Marek, T. \& Maslach, C. 1993. Professional Burnout. Washington: Taylor \& Francis.

SiLES, J. 1999. Historia de la enfermeria. Alicante: Aguaclara.

SMALL, E. 1996. Valuing the emotional labour of nursing. Nursing times. 91(26):40-1.

SMITH, P. 1988. Recruit and retain. The emotional labour of nursing. Nursing times. 84(44): 50-1.

SMITH, P. 1989. Nurse's emotional labour. Nursing times. 85(47): 49-51.

SMITH, P. 1991. The nursing process: raising the profile of emotional care in nursing training. Journal of advanced nursing. 16(1): 74-81.

SMith, P. 1992. The emotional labour of nursing. London: Macmillan

Staden, H. 1998. Alertness to the needs of others: a study of the emotional labour of caring. Journal of advanced nursing. 27(1): 147-56.

Walster Hatfield, E., Cacciopo \& Rapson. 1994 Emotional contagion. Cambridge: Cambridge University Press.

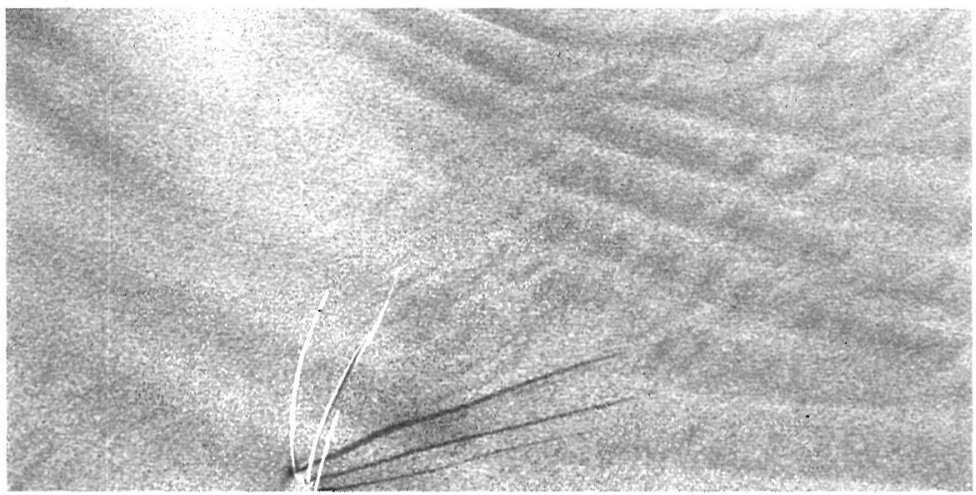

